\title{
Quadrature Slotted Surface Coil Pair for Magnetic Resonance Imaging at 4 Tesla: Phantom Study
}

\section{Arreglo de un par de antenas superficiales en cuadratura con ranura circular para imagenología por resonancia magnética a 4 Tesla: estudio con fantoma}

\author{
Solis S.E. \\ Departamento de Ingeniería Eléctrica \\ Universidad Autónoma Metropolitana Iztapalapa, México, DF \\ Correo:solisnajera@gmail.com \\ Tomasi D. \\ Medical Department \\ Brookhaven National Laboratory, Upton, N.Y. 11973, USA. \\ Correo:tomasi@bul.gov
}

\author{
Hernandez J.A. \\ Departamento de Ingeniería Eléctrica \\ Universidad Autónoma Metropolitana Iztapalapa, México, DF \\ Correo:jahz.ingbiom@gmail.com \\ Rodriguez A.O. \\ Departamento de Ingeniería Eléctrica \\ Universidad Autónoma Metropolitana Iztapalapa, México, DF \\ Correo:arog@xanum.uam.mx
}

Información del artículo: recibido: agosto de 2009, reevaluado: octubre de 2010, aceptado: enero de 2011

\begin{abstract}
A coil array was composed of two slotted surface coils forming a structure with two plates at $90^{\circ}$, each one having 6 circular slots and is introduced in this paper. Numerical simulations of the magnetic field of this coil array were performed at $170 \mathrm{MHz}$ using the finite element method to study its behaviour. This coil array was developed for brain magnetic resonance imaging to be operated at the resonant frequency of $170 \mathrm{MHz}$ in the transceiver mode and quadrature driven. Numerical simulations demonstrated that electromagnetic interaction between the coil elements is negligible, and that the magnetic field showed a good uniformity. Phantom images were acquired with our coil array and standard pulse sequences on a research-dedicated 4 Tesla scanner. In vitro images showed the feasibility of this coil array for standard pulses and high field magnetic resonance imaging.
\end{abstract}

\section{Keywords}

- coil array

- magnetic resonance imaging

- electromagnetic simulation

- slotted surface coil

- transceiver

- quadrature-driven 


\section{Resumen}

Se presenta un arreglo de antenas transceptoras operado en cuadratura, se construyó con dos antenas superficiales con 6 ranuras circulares y separadas por un ángulo de $90^{\circ}$. El campo magnético de este arreglo de antenas se simuló de manera numérica a la frecuencia de $170 \mathrm{MHz}$, empleando el método de elemento finito. El arreglo de antenas se desarrolló para aplicaciones de la imagenología del cerebro por resonancia magnética a la frecuencia de $170 \mathrm{MHz}$. Las simulaciones numéricas mostraron que la interacción electromagnética entre los elementos es nula, y muestra una buena uniformidad de campo. Se generaron imágenes de fantoma con el arreglo de antenas, usando secuencias de pulsos estándar con un sistema de 4 Tesla dedicado para hacer investigación. Las imágenes en vitro muestran la factibilidad de este tipo de arreglos de antenas con secuencias de pulsos estándar para imagenología por resonancia magnética de altos campos.

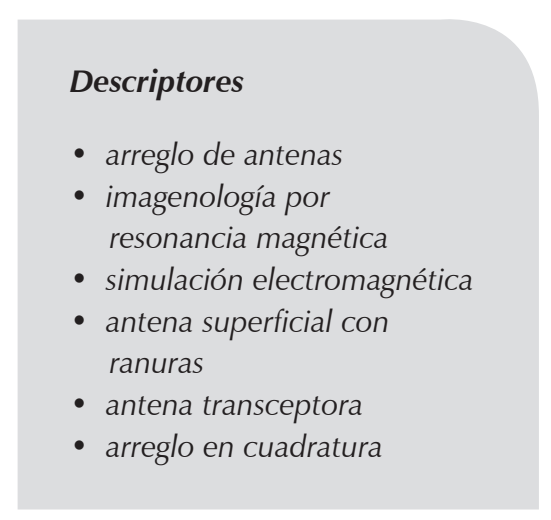

\section{Introduction}

Multi coil magnetic resonance imaging (MRI) has quickly become a wide spread commodity since its reduced acquisition times (> $8 \mathrm{~ms}$ ). The developments in RF-technology and multi-coil arrays, which have been largely triggered and influenced by the challenges of high field MR have led to quite a dramatic improvement of clinical 1.5 and 3T systems (Henning, 2008). The quadrature coil pair has been widely accepted for magnetic resonance imaging applications due to their high signal-to-noise ratio (SNR) over large volumes of interest. This coil array compromised of independent coil elements forming an orthogonal structure is called a quadrature RF coil pair (Chen et al., 1983). The principal radiofrequency (RF) magnetic field components are orthogonal or nearly orthogonal, such that they can directly receive the circularly polarized MRI signals from a sample. Quadrature surface MRI detectors usually comprised of circular loop. However, the non-uniform sensitivity profile is the main disadvantage of surface RF coils compared to volume coils, because it produces marked decreases of signal-to-noise ratio (SNR) for increasing coil-tissue distances. Kumar and Bottomley recently published an optimised version of the quadrature surface coil pair (Kumar, 2008). Our group has recently introduced a new surface coil with a significant improvement in image SNR over the circular-shaped coil (Rodríguez, 2006), the so-called magnetron surface coil (Solis et al., 2006). In this paper, a modified design of the magnetron surface coil composed of circular slot was used to construct a quadrature slotted surface coil pair. Then, a prototype of an array of 2 slotted surface coils was developed to perform in vitro MR imaging experiments at 4 Tesla. Phantom images were then acquired using standard spin echo sequences and showed good quality.

\section{Method}

Magnetic field numerical simulation

To predict image quality, the spatial distribution of the magnetic field, B1 produced by the slotted surface coil pair was numerically simulated using a finite element method (FEM) with a three-dimensional electromagnetic model. Figure $1 \mathrm{~b}$ shows a schematic of the coil pair and the spherical phantom used to mimic the salinesolution phantom of the imaging experiments. The FEM was used due to its ability to model complex geometries with acceptable accuracy. All numerical computations were carried out with the commercial software tool, FEMLAB (COMSOL, Burlington, MA, USA) in a standard Intel PC running Windows OS. The simulation parameters were; mesh element number: 1308, degrees of freedom: 83595, and solution time: $92.62 \mathrm{~s}$.

\section{Quadrature coil pair prototype}

Two slotted surface coils with equal dimensions (figure 1c) were machined in copper sheets to form ellipses with 6 circular slots each. These two surface coils were mounted on acrylic bases with a $90^{\circ}$ separation. Figure 1a shows an illustration of the coil array configuration. $50 \mathrm{Ohm}$ coax cables were attached to each coil to transmit the RF signal and to receive the MR signal coming from the phantom into the scanner. The coil pair was driven in the quadrature mode. To tune and match the coils, both nonmagnetic fixed-value capacitors $\left(\mathrm{C}_{\mathrm{D} 1^{-}}\right.$ $\left.\mathrm{C}_{\mathrm{D} 4}\right)$ and trimmers $\left(\mathrm{C}_{\mathrm{t} 1}, \mathrm{C}_{\mathrm{t} 2}, \mathrm{C}_{\mathrm{m} 1}\right.$ and $\left.\mathrm{C}_{\mathrm{m} 2}\right)$ were used and evenly distributed around the coils. Each slotted coil was tuned to $170 \mathrm{MHz}$ (proton frequency) and $50 \mathrm{Ohms}$ matching was used to assure maximum energy transfer. To reduce coupling between the two coil elements a 

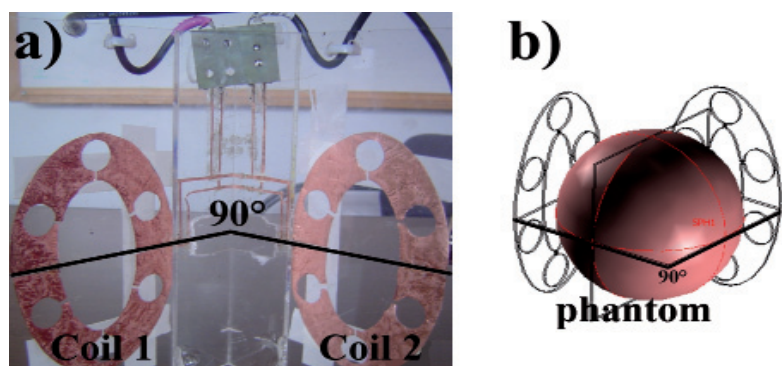

specially designed circuit was developed and shown in figure 2 . The resonant frequency was measured using a network analyzer (Model 4396A, Hewlett Packard, Agilent Technologies, $C A)$ as the loss return $\left(\mathrm{S}_{11}\right)$. The quality factor $(Q)$ of the coil pair prototype was experimentally determined by measuring the resonant frequency of the coil pair divided by the 3-dB bandwi$\mathrm{dth}, \Delta \omega$, with quarter-wavelength coaxial cables at the input of each coil. The loaded $Q$-value was measured while the coil was placed on the top of a cooking oil-filled phantom. The resonant frequency of the circularshaped coil was measured following the same procedure as above.

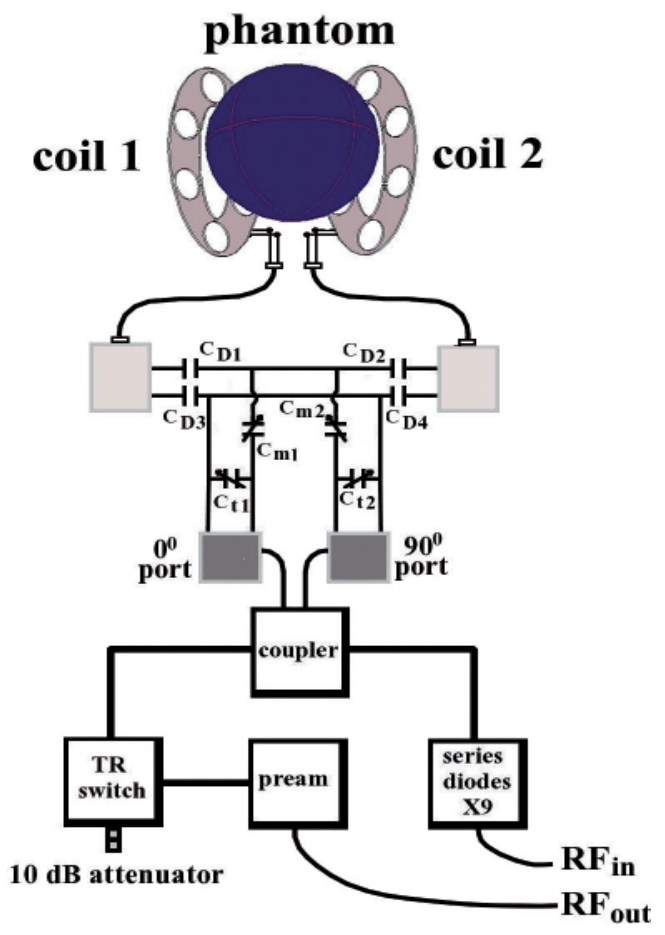

Figure 2. Capacitive decoupling circuit for the magnetron coil pair: cable $0^{\circ}$ and $90^{\circ}$ represent coil element 1 and coil element 2, respectively. Capacitors $\mathrm{C}_{\mathrm{d} 1}$ and $\mathrm{C}_{\mathrm{d} 2}$ do the decoupling of both coils. $C_{m 1}$ and $C_{t 1}$ are nonmagnetic trimmers (1-30 pF) for tuning and $50 \Omega$ matching for coil 1 , and similarly $\mathrm{C}_{\mathrm{d} 2}$ and $\mathrm{C}_{\mathrm{t} 2}$ for coil 2

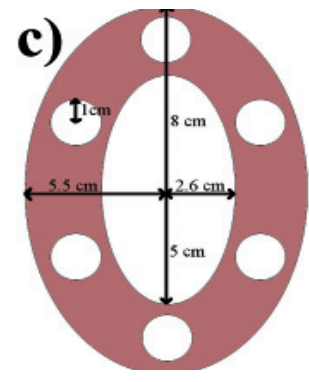

Figure 1. a) Quadrature coil pair prototype formed with two slotted surface coils mounted on an acrylic sheet bent $90^{\circ}$, b) Eagle view of the 3D model showing coil array and phantom and c) prototype's dimensions

\section{The probe interface}

In the MRI system the signal is usually processed in two separate paths. To obtain the magnetic resonance (MR) response from the sample (biological tissue), it is excited by an RF signal that is transmitted with the coil first (when driven with a voltage at a particular frequency, generates a current distribution that produces a B1 field over a sample). After switching off the excitation, the sample response is detected with the reception coil.

The pre-amplifier. The outgoing signal's response from the measured sample is usually very weak and must be amplified by a low noise pre-amplifier. This is the first stage of amplification. The pre-amplifier, is a device specifically designed to have a good noise characteristics and it may be within the magnet.

Impedance matching network. The input impedance of RF resonator needs to match $50 \Omega$ cable. Simple LC circuit is divided into two types, i.e., serial resonator and parallel resonator. The impedance of ideal parallel resonator is infinitely big, while of ideal serial or pure parallel resonator is nearly zero. Both pure serial or pure parallel resonator can not absorb efficiently RF power because of power reflected. The impedance of the parallel resonator can be reduced by adding a capacitor in series. It is possible to reduce to $50 \Omega$. The impedance of serial resonator can be elevated through adding a capacitor in parallel. It is also possible o raise to $50 \Omega$. The RF power can be delivered from transmitter to RF coil without reflecting only under impedance matching condition.

TR switch. The RF switch is used to separate the incoming and outgoing signals of the combined RF coil. The combined RF coil can be used to receive and transmit the signal simultaneously, so there are needs to choose different paths for the transmitted and received signals because of their different processing. When transmitting, the signal coming into the RF coil is amplified up to the power of $1 \mathrm{~kW}$ to excite the measured sample. Owing to a different signal levels and the different signal processing techniques used, the signals must be separated from each other with high switching 
times, typically a few microseconds (time ranges from 1 to $5 \mu \mathrm{s})$.

The coupler (phase shifter). The phase-sensitive detection ('in quadrature') reduces the Larmor frequency to a manageable value from a computational point of view. The phase of the reference may be adjusted as desired, before the reference is split of $90^{\circ}$. The two products are filtered to remove the high-frequency components and two quadrature signals are ready.

\section{In vitro MRI experiments}

To test the validity of this coil design, phantom images were acquired with a spherical phantom. All imaging experiments were performed on a 4 Tesla whole-body Varian/Siemens system (Varian, Inc, Palo Alto, CA) interfaced with an INOVA console. T1-weighted axial images of a spherical phantom were acquired with the following acquisition parameters: TE/TR $=20 / 1000 \mathrm{~ms}$, FOV $=20 \times 20 \mathrm{~cm}$, matrix size $=256 \times 256$, slice thickness $=10 \mathrm{~mm}, \mathrm{NEX}=1$.

\section{Results and discussion}

Numerical simulations were performed using a spherical phantom to mimic the human brain and shown as bi-dimensional images in figure 3 right. The numerically computed magnetic field clearly shows that no magnetic interaction between the coil elements is present. This is mainly due to the separation of the coil elements. These results confirm a well-known fact that coils orthogonally arranged show no mutual interaction. The typical attenuation of the field generated by the coil elements for points far away from the coil plane can also be observed.

The spherical phantom image in the figure 3 left shows a remarkable agreement with the numerical simulations: no interaction between coils and the characteristic signal attenuation are shown. This also implies that a good isolation of the two channels was able to

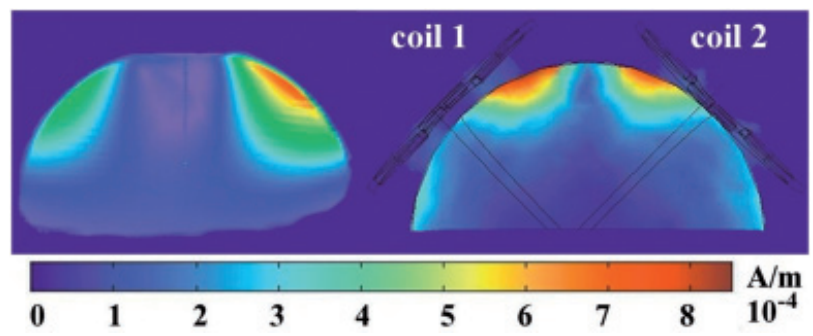

Figure 3. Image comparison: phantom image (left) [arbitrary units] and numerical simulation (right) $\left[\mathrm{A} / \mathrm{m}^{2}\right]$ of the coil array achieve with the figure 2 circuit. The measured coil quality factors are: 15.45 at $-22.5 \mathrm{~dB}$ for coil element 1 , and 18.88 at $-24.3 \mathrm{~dB}$ for coil element 2 . These calculations are referred to the unloaded case. When the spherical phantom was placed in the coil array (loaded case), the quality factors were reduced around $30 \%$ for both coil elements. The experimental measurement of the resonant frequency is shown in figure 4 for both channels.

These data prove that both coil elements are at the same resonant frequency and that their expected performances should be practically the same. The $(\mathrm{dB})$ attenuation is also within the acceptable range for surface coil commonly used at high frequency. Coupling of the coil elements can appreciated when there is a widening of the spectra in figure 4 . This implies that the individual coil performance shows a low quality factor. This is particularly important since at resonant frequency values greater than $100 \mathrm{MHz}$ the coil element isolation in an array is achieved at expense of low coil performance. However, the figure 2 circuit is able to decouple the two coils to produce a great deal of isolation between them since the widening is only a few $\mathrm{MHz}$.

From these data it can be appreciated that the quality factors are in good agreement with those reported in the literature (Kumar, 2008). Additionally, the capacitive circuit of figure 2 plays an important role in the coil performance reflected in the good quality factors mentioned above and the loss return profiles of figure 4 .

An example of an axial image taken at the midsection of the coil array is shown in figure 5a. Axial images were also obtained with a saline solution phantom and the slotted-coil array. Figure 5b shows a T1-weighted image acquired with a standard spin echo sequence. Despite that only the magnetic field was numerically computed, there is a good agreement between the numerical results and the actual phantom image.

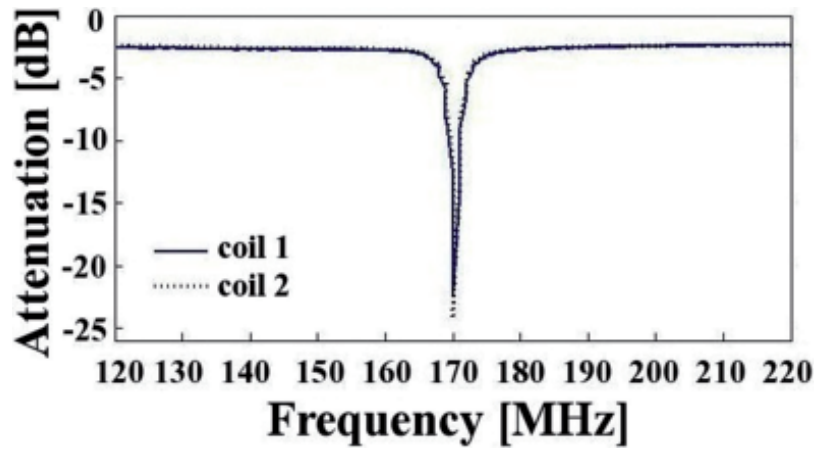

Figure 4. Plots of loss-return-vs.-frequency for both coil elements for the unloaded case. coil 1 and represent the $0^{0}$ channel and $90^{\circ}$ channel, respectively 


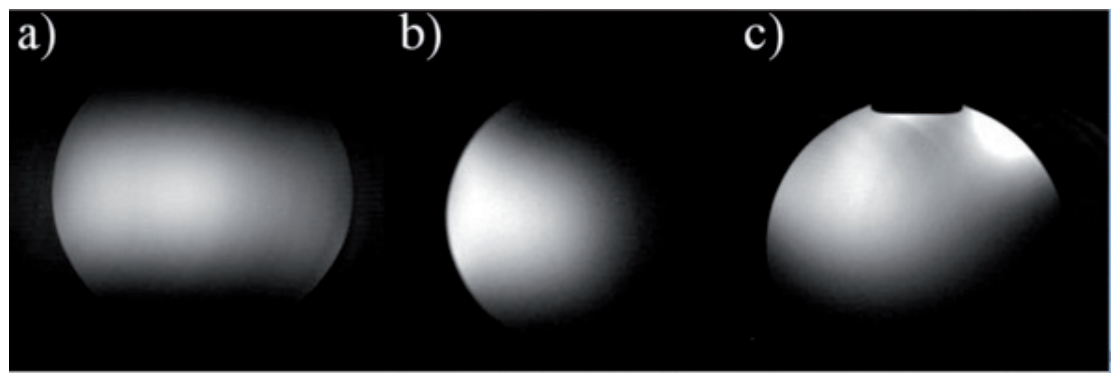

Figure 5. Phantom images acquired with our coil array in different orientations: a) coronal, b) saggital and c) transversal

A more realistic comparison should include the computation of the signal-to-noise ratio in the form of bi-dimensional images. This electric magnetic field generated by the sample to be imaged should necessarily be determined too. Phantom images showed a good image SNR. Field uniformity still requires improvement to fully cover the phantom sphere. A new configuration is necessary to be used to actually cover the sphere in more adequate fashion. This may be solved by rearranging the position of the two coils via the angle formed by them. It is suggested to use angle value lower than $90^{\circ}$ without reducing the volume available to accommodate the spherical phantom. These results confirmed the viability of this coil array design and its compatibility with high field imagers and standard pulse sequences. This coil array may also prove to be of some use for applications with parallel imaging techniques.

\section{Conclusions}

It has been show that the slotted surface coil can be used to form a coil array to acquire phantom images with a research dedicated magnetic resonance imager. It has also been proved that the coil array design is fully compatible with standard pulse sequences usually used in the clinical environment.

\section{Acknowledgments}

S. E. S. thanks the Laboratory Directed Research and Development from U.S. Department of Energy (OBER) and, the National Council of Science and Technology of Mexico (CONACyT) for a Ph. D. scholarship.

\section{References}

Chen C., Hoult D.I., Sank V.J. Quadrature Detection Coils- a Further $\sqrt{2}$ Improvement in Sensitivity. Journal Magnetic Resonance, 54: 324-327. 1983.

Hennig J. Ultra High Field MR: Useful Instruments or Toys for the Boys? Magn. Reson.Mater. Phy, 21:1-3. 2008.

Kumar A., Bottomley P.A. Optimized Quadrature Surface Coil Designs. Magn. Reson. Mater. Phys., 21:41-52. 2008.

Rodriguez A.O. Magnetron Surface Coil for Brain MR Imaging. Arch. Med. Res., 37:804-807. 2006.

Solis S.E., Tomasi D., Rodriguez A.O. Slotted Surface Coil for Magnetic Resonance Imaging at 4T, 13th Ann. Meet. ISMRM, 2006, 2612 p. 


\section{About the authors}

Sergio E. Solis. Received his Ms. Sc. degree (biomedical engineering) in 2003 from the Universidad Autonoma Metropolitana-Iztapalapa, then continued his research work towards a gained his Ph.D. degree in high field magnetic resonance imaging in 2010 from the same university. He is engaged in the development of novel designs of RF coils for various applications raging from animal models to study human diseases. He is currently doing a posdoc on BOLD fMRI of pain at the National Institute of Psichiatry of Mexico.

Dardo Tomasi. Obtained his Ph.D. in Physics from the University of Buenos Aires in 1996. He joined the Medical Department of Brookhaven National Laboratory in 2001 as research associate. He focuses his research interests on the development of advanced MRI techniques to study the effects of brain diseases and psycho stimulants on brain activation. He is also interested in developing functional MRI techniques with high spatial and temporal resolution, and evaluating the effect of MRI acoustic noise on cognition, particularly attention and memory.

Jorge A. Hernandez. Studied Biomedical Engineering (B. Sc. degree, 2008) at the Universidad Autonoma Metropolitana-Iztapalapa. He worked on the electromagnetic simulations of various coil designs to study their performance. He also built a volume coil for whole-body imaging of rodents at 7 Tesla. He is currently working for Bruker Mexicana as a service engineer dealing with various technical aspects of NMR and optical spectrometers.

Alfredo O. Rodriguez. Earned his B. Sc. degree in physical engineering from Universidad Autonoma Metropolitana-Atzcapotzalco 1990, Mexico, and his Ph.D. degree in Physics (MRI) in 1997 from the University of Nottingham, Britain. There he worked under the supervision of Sir Peter Mansfield in RF coil developing and flow imaging. He joined the Department of Electrical Engineering of the Universidad Autonoma Metropolitana-Iztapalapa, Mexico in 1998. He is co-founder of the first CONACYT-funded national laboratory on medical imaging. His scientific interests include development of RF coils for MRI applications, flow MR imaging particularly turbulent flows in the cardiovascular system, and MRI with remote detection. 